\title{
Espacio público y vida cotidiana: hacia un cambio de teo- rías en diseño urbano y arquitectónico.
}

\author{
Public space and daily life: towards a change of theories in urban and architectural \\ design.
}

Recibido: Mayo 2018

Aceptado: Agosto 2019

Javier Fuentes Farías ${ }^{1}$

\section{Resumen}

El espacio urbano se encuentra vinculado a cuestiones sociales, culturales, político-económicas, y también epistémicas y filosóficas, por lo cual éstas son áreas de interés para el diseño. Planteo que no es posible conocer dichas cuestiones sin antes revisar el sentido que damos a los conceptos que permiten incluir el punto de vista de la gente, entre ellos: espacio público y mundo de vida, identidad cultural, imaginarios urbanos, o representaciones sociales. Examinaremos dichos conceptos bajo la idea de su interdefinición, es decir, que para explicarlos debemos recurrir a otras conceptualizaciones, y que al hacerlo la arquitectura y filosofía se encuentran. Podemos confirmar ciertas dificultades en su estudio, tales como su carácter subjetivo, es decir, su inaccesibilidad al método científico, donde observamos algunas teorías que, como el posmodernismo y el posestructuralismo, ofrecen nuevas pistas desde la fenomenología y las ciencias cognitivas. Otros ejemplos provienen de la geografía constructivista y la llamada Teoría Social. En esa línea, concluimos que los especialistas en diseño interpretan el punto de vista de las personas mediante su propia experiencia como habitantes de la ciudad.

\section{Palabras Clave:}

Cognición social; Fenomenología; Posmodernismo.

\begin{abstract}
Urban space is linked to social, cultural, politicaleconomic, and also epistemic and philosophical issues, according to which design discipline need to face it. I argue that it's not possible to know these questions without first reviewing some key concepts, such as urban culture, social representations, identity, and others coined to seek for people's mind, interacting, and dwelling, including public space and life-world. But to define these key concepts we need another conceptual categories, so in that way architecture and philosophy meets. Those examples demonstrate the current problem about the scientific status of people's inner world or subjectivity, as in postmodernism, post-structuralism, and deconstructivism. Rather than explain this, I will summarize some theories that offer new clues from phenomenology and cognitive sciences, such as constructivist geography and so-called social theory. In this line, we conclude that design specialists interpret people's point of view through their own experience as urban inhabitants.
\end{abstract}

\section{Keywords:}

Social cognition; Phenomenology; Posmodernism.

\footnotetext{
${ }^{1}$ Nacionalidad: mexicano; adscripción: Universidad Michoacana de San Nicolás de Hidalgo; fuentes88@hotmail.com
} 


\section{Introducción.}

El presente trabajo forma parte de una línea de investigación del autor de este escrito, acerca del espacio arquitectónico y sus aspectos simbólicos y epistémicos, tema que, según recientes hallazgos, atañe a distintas disciplinas científicas y humanísticas. En ésa temática he llamado la atención ${ }^{2}$ sobre el espacio construido y la problemática acerca del papel de las personas los agentes, o actores urbanos- en los procesos constructivos y de diseño.

Entre otros temas específicos, he planteado antes el interés por la arquitectura moderna y posmoderna en la medida que manifiestan una nueva tradición, es decir, un cambio de teorías y conceptos acerca de las categorías y concepciones fundamentales del quehacer arquitectónico. La importancia de la arquitectura moderna puede valorarse en la medida que se plantea nuevos retos, ya sea desde la necesidad de nuevas herramientas de diseño urbano y arquitectónico, como de afrontar la creciente complejidad de los procesos constructivos y los nuevos materiales disponibles. Al mismo tiempo la figura humana cobró gran importancia y surgió el interés por los espacios públicos de las nuevas ciudades.

Lo que Giedion (2009) llama una nueva tradición, corresponde a la idea de que "el objetivo de la arquitectura ya no es la forma independiente y sin relación, sino la organización de formas en el espacio: la concepción del espacio". (p. 21) Esa nueva concepción se refiere, en suma, a la necesidad de "salvar la brecha entre pensamiento y sentimiento" (Norberg-Schulz, 2005: 249), mediante la construcción de un nuevo lugar, un espacio significativo para la existencia humana, para habitar.

En otros escritos he planteado esta problemática, no sin señalar su importancia en las disciplinas del diseño. Una premisa en el presente trabajo es que este tipo de conocimiento acerca de las subjetividades humanas, o sea de la cultura simbólica y las interacciones sociales, es indispensable para que quienes diseñan el espacio construido tengan dicha perspectiva para así poder integrarla en los procesos de diseño. Por ello, trataré dicha cuestión, que es el punto de vista de las personas, o el punto de vista subjetivo o cualitativo, expresado en los espacios públicos de las nuevas urbanizaciones de la posguerra, mediante la categoría conceptual de "mundo vivido", o "vida cotidiana", según la Teoría Social y los estudios urbanos contemporáneos. (Giddens, 2006; Lindón, 2014, 2012; López y Tena, 2015; Duhau y Giglia, 2007; López, 2008, 2014, 2015)

Este tema lo abordé antes en otros escritos donde revisé algunos conceptos clave empleados en distintas disciplinas interesadas en la relación entre el espacio construido y las personas que lo habitan, conceptos como identidad urbana, cultura, imaginarios urbanos, representaciones sociales, "espíritu del lugar", subjetividad, agencia social, habitabilidad, espacio existencial, y otros.

Entre otros resultados teníamos que, entre otros cambios fundamentales, el objeto arquitectónico dejó de pensarse como algo separado de su contexto, y al mismo tiempo se cuestionó la idea de los objetos como contenidos en un espacio neutro e inmutable. Es decir, cambiaron las concepciones o categorías conceptuales fundamentales en arquitectura, como el espacio y el objeto arquitectónico. Así por ejemplo, con movimientos como el posmodernismo tenemos un creciente interés por el contexto de la obra arquitectónica, por las relaciones entre dicho objeto y los demás objetos construidos circundantes, y por los significados de los lugares públicos y del todo construido.

En suma, pueden mencionarse dos puntos importantes: uno, el cambio de las concepciones tradicionales de la arquitectura, de "aquello que se creía eran las persistencias en arquitectura: sujeto y objeto, figura y fondo, sólido y vacío, y las relaciones de la parte con el todo". (Eisenmann, 2011: 16) Y dos: "el gran giro epistemológico, el cambio de concepciones y teorías acerca del ser humano, de la cultura, lo simbólico, y lo subjetivo, como objetos de interés surgidos al mismo tiempo en distintas disciplinas científicas y humanísticas.

Así, en la siguiente sección aportaré algunos elementos con el fin de mostrar que, en primer lugar, comprender y explicar nuestro problema de estudio -la relación entre espacio público y vida cotidiana- requiere un cambio de teorías, sustentado a su vez en una crítica y elucidación conceptual, lo que podemos apreciar en los ejemplos incluidos

\footnotetext{
${ }^{2}$ Citaré artículos de mi autoría publicados en revistas indexadas, o inéditos, en proceso de dictamen por revistas arbitradas e indexadas.
} 
aquí: las categorías conceptuales de espacio público y vida cotidiana. Dicho cambio tiene como características la síntesis o fusión de horizontes disciplinares, entre ciencias y filosofía, y entre distintas disciplinas que se plantean compartir un enfoque constructivista como el de la epistemología genética de Jean Piaget, el cual es correlativo a los puntos de vista en los ejemplos mencionados.

En una segunda sección presento algunos aspectos del tema general ya descrito, mediante una comparación con otros conceptos clave acuñados de manera correlativa al surgimiento de nuevas corrientes filosóficas, científicas, y humanísticas (algunos ya mencionados), enfocadas al estudio de la subjetividad humana en general, un tema que parecía tabú desde el pensamiento científico, y que en ciencias sociales se planteó como un cuerpo teórico-conceptual llamado Teoría Social, bajo nuevas consideraciones sobre la teoría del conocimiento y sobre la complejidad creciente de los procesos de urbanización. Como ejemplo tenemos los llamados "giros" o cambios en los fundamentos teóricos del pensamiento urbanístico y los estudios urbanos, las ciencias sociales y la geografía humana, entre otras disciplinas.

El problema ha sido que toda experiencia subjetiva resulta inaccesible al pensamiento cartesiano, y que en tal caso resulta conveniente echar mano de los paradigmas hermenéutico y fenomenológico, ampliamente referidos en la teoría social y en recientes aportes a la teorización del diseño urbano y arquitectónico.

Así, antes de examinar directamente el espacio público y el mundo de vida de las personas, parece necesario esclarecer el sentido de cómo acordar criterios entre las distintas disciplinas, empezando por arquitectos, historiadores, y sociólogos, para asegurar que efectivamente hay un conocimiento objetivo acerca de las distintas vertientes de los fenómenos y procesos urbanos señalados como correlacionados con el espacio público y con quienes habitan la ciudad. Por ello, planteo que una revisión y crítica de las mencionadas nociones fundamentales en diseño urbano y arquitectónico permitiría tener una visión de conjunto de las principales ideas prevalecientes en cuanto a la correlación antes señalada.

Concluiremos señalando que, en materia de teoría, crítica, e historia de la arquitectura, hemos visto un cambio conceptual y metodológico necesario para comprender y diseñar el espacio construido, entre ellos la idea del espacio como una totalidad que incluya sus aspectos simbólicos y sociales. Así, emprender el estudio de los lugares públicos en la actualidad, desde distintas perspectivas científicas y humanísticas, permite al especialista en diseño comprender e interpretar el punto de vista de quienes habitan el espacio construido.

\section{La Posmodernidad y las nuevas reglas del diseño.}

En escritos anteriores he abordado el tema de la llamada Nueva Tradición en arquitectura, periodo histórico que abarca el Art Noveau, la arquitectura moderna, y el posmodernismo, tema cuya complejidad ha requerido ampliar las fronteras de la arquitectura para abrazar los paradigmas de la fenomenología, hermenéutica, y de los estudios de la complejidad. (Montaner, López,

Como mencioné arriba, los cambios ocurridos a partir del periodo moderno se resumen como un cambio de conceptos y teorías en el diseño urbano y arquitectónico, toda vez que, en la posguerra, se presentaban retos como la construcción de nuevos asentamientos y nuevos edificios, en los cuales la figura humana jugaría un papel preponderante, lo cual cobró el interés de distintas disciplinas hacia los procesos urbanos.

Los cambios en las concepciones fundamentales de la arquitectura, reflejados en una nueva tradición, son correlativos a "un gran cambio de paradigma epistémico caracterizado por la idea de que conocimiento y lenguaje son interdependientes, y de que ante una realidad compleja y cambiante se requiere un enfoque interdisciplinar y transdisciplinar". Esta ha sido una de las premisas que he sostenido anteriormente, por lo cual se argumenta aquí la importancia metodológica de una revisión y crítica de conceptos y teorías acerca del diseño urbano y arquitectónico.

Entre otros ejemplos he señalado el deconstructivismo (la Deconstrucción en arquitectura) "como una actividad o un proceso mediante el cual tomamos conciencia de los límites del lenguaje y del pensamiento". (Davies, 2011: 37) Este autor arguye que solo podemos comprender el mundo "por medio del lenguaje y otros sistemas de signos"; ya que para hablar 
de los signos se requieren otros signos, y para hablar de éstos se requieren a su vez de otros, se vuelve un juego de espejos, "una cadena sin fin". Por ello no puede darse por sentado la existencia de una realidad fija e inmutable, "a la que el lenguaje se pueda referir".

Aquí es evidente el cuestionamiento de una base teórica que proviene del reduccionismo cientificista, por llamarle así al paradigma de la ciencia cartesiana y el positivismo lógico. Ante ello, hacia mediados del siglo anterior surgen nuevas categorías conceptuales como las de sistema, emergencia, autoorganización, hologramaticidad, retroalimentación, y otras que confirman la idea de Montaner acerca de la muerte del objeto arquitectónico y el surgimiento de un objeto complejo.

Entre otros ejemplos específicos de influencias en arquitectura tenemos el posestructuralismo, cuyas premisas se basan en que el espacio construido es como un lenguaje, y éste a su vez es un sistema complejo, una totalidad cuyos significados hay que buscarlos en la relación entre las partes y el todo, y, en cuanto al diseño urbanoarquitectónico, entre la morfología urbana, las formas construidas, y aquellos cuyas vidas transcurren cotidianamente en dicho espacio. Tal relación puede observarse claramente cuando dicho espacio manifiesta funciones simbólicas y sociales, por ejemplo en el caso del espacio público, término con el cual nos referimos a las calles, plazas, jardines, y otros elementos que forman la traza urbana, en los cuales transcurre la vida cotidiana de los habitantes de la ciudad.

Podemos ver cómo en el pensamiento urbanístico contemporáneo, y en general, en los estudios urbanos, se ha planteado la necesidad de elaborar nuevas preguntas, ya que: "en el conocimiento de las determinaciones del diseño juega un papel fundamental el esclarecimiento de los actores sociales involucrados en el proceso de diseño, ya que es evidente la naturaleza sociohistórica de éstos" (López, 2014: 24).

Así pues, en anteriores escritos se concluyó que:

la complejidad y diversidad de aspectos a considerar tanto en los procesos de diseño y construcción del espacio habitable, como del instrumental o bagaje teórico y conceptual necesario para investigar y comprender tales procesos, es dificil de abordar sin antes emprender una crítica de las categorías tradicionales, empezando por el espacio construido y sus implicaciones en lo social e histórico.

Concluimos también que en cuanto a las categorías conceptuales ya mencionadas, aquellas referentes a la subjetividad de los actores urbanos, tales como cultura e identidad, representaciones sociales, imaginarios sociales, etc., podían entenderse como como procesos de producción e interpretación de signos, símbolos, y significados, y finalmente como procesos cognitivos de percepción, categorización, conceptualización, e interacción social.

Como resultado de tales observaciones, en particular sobre el problema de la subjetividad y el papel de los individuos en la sociedad, se concluyó también que era necesario "ir al fondo de la problemática sobre el estatuto científico del habitar humano, (Lindón) y sus repercusiones en la filosofía y las humanidades", pero también en la configuración de nuevas corrientes de investigación como las llamadas ciencias cognitivas, y otras citadas aquí, que otorgan "una creciente validez" a los paradigmas de la fenomenología y hermenéutica.

Por ello señalé antes, siguiendo a López Rangel la necesidad de

...valorar la influencia de la hermenéutica, la fenomenología, la semiótica, y la epistemología misma, en los estudios urbanos y el pensamiento urbanístico en general, y proceder a elaborar un cuerpo teórico interdisciplinar, empezando por la construcción de los procesos urbanos en términos de sistemas complejos, lo cual no es una tarea científica sino filosófica.

Dicho cambio puede resumirse como una crítica al modelo del conocimiento que proviene del empirismo lógico, del dualismo cartesiano, y de la racionalidad científica, cuyos aspectos principales ya puntualizaron Piaget y García. (García, 2006)

En cuanto al espacio público como objeto de estudio, ha motivado infinidad de enfoques y teorías, tanto en arquitectura y urbanismo como en otras disciplinas interesadas en la subjetividad de los actores urbanos, entre ellas la antropología y las ciencias sociales, la psicología social, la geografía humana.

Recordemos que:

...en la vida más en general, está por un lado el andamiaje externo - el lugar físico o la arquitectura, el juego, las reglas, o simplemente las costumbres-, y por el otro, mis capacidades 
corporizadas y cognitivas: capacidades que comienzan y continúan siendo sensoriomotoras, perceptivas, y orientadas a la acción". (Gallagher, 2014: 243)

La cita de Gallagher, como ejemplo de las nuevas corrientes interdisciplinares que estudian el espacio habitado, permite argumentar que es tal la complejidad del tema en cuestión lo que hace de la disciplina del diseño una especialidad que linda con la filosofía, las ciencias sociales, y otras corrientes interdisciplinares como las ciencias cognitivas, la fenomenología sociológica, y la geografía constructivista.

Un estudioso del espacio público como Jordi Borja, en el prólogo a "Identidad y Espacio Público" (Sánchez y Domínguez, Coords.; 2014: 11), señala que desde su dimensión urbanística el espacio público debe ser considerado "como la base estructural de la ciudad", esto es, debe considerarse el factor principal de ordenamiento en términos de calidad ambiental, y de la imagen, accesibilidad, movilidad, y transición entre espacio público y privado.

En otra observación (Íd.: 13), Borja indica que "el concepto de espacio público es multidimensional, puede abarcar aspectos muy abstractos tratados desde la filosofía y la teoría social, política y jurídica." Al mismo tiempo tenemos el interés sobre distintos aspectos de la ciudad expresados mediante dicho concepto, ya sea sobre determinados lugares, barrios, esquinas, y su relación con colectivos sociales, por parte de disciplinas como geografía, antropología, etnología, sociología, psicología, e incluso el periodismo, agrega Borja. Y todo ello atañe a las especialidades de urbanistas, planificadores, arquitectos, y científicos sociales "interesados en la relación entre las formas urbanas y los usos sociales". (Ib.: 14)

En otros ejemplos mencioné antes el trabajo del Dr. López Rangel, para quien es necesaria una re-conceptualización de los nuevos tipos de desarrollos urbanos surgidos durante las últimas cinco o seis décadas, también hace imprescindible plantear nuevas preguntas y nuevas problemáticas, formular nuevas hipótesis, e innovar métodos y modelos de análisis (Cfr. López Rangel, en el prólogo a López y Tena, 2015: 7).

Tal preocupación es compartida en distintas líneas de investigación acerca de las prácticas socio-espaciales o metropolitanas (Cfr. Duhau y Giglia, 2008: 47, 437, 536), es decir, aquellas que "tienen que ver con la vida cotidiana", bajo "ciertas pautas recurrentes en la forma de usar y vivir la metrópoli." Algunos ejemplos provienen de la geografía constructivista (Lindón, Claval, Debarbieux), y de la mencionada Teoría Social, donde tenemos la fenomenología sociológica de Gallagher y otros contemporáneos, además del llamado Pensamiento Urbanístico Contemporáneo (López, ), donde citamos a Tena, Duhau y Giglia, Domínguez, etc.

\section{El punto de vista subjetivo y el enfoque fenomenológico.}

En el ámbito del diseño resulta que no podemos separar los aspectos formales de los cualitativos, es decir, aquellas cuestiones de estructura, tectónica, simetría, lleno y vacío, y otras de carácter espacial y compositivo, con asuntos más complejos como los sociales, políticos, estéticos, etc. De tal modo estos conceptos, necesarios para el desarrollo del diseño formal, se encuentran relacionados e interdefinidos con otros de carácter cualitativo.

En otros ejemplos provenientes de la teoría social "el interés explícito por el sujeto" (Lindón, 2012: 606) se replantea como el interés por un "sujeto habitante", colocado "en el meollo de la cuestión", un sujeto inserto en un mundo social y cultural, pero además vinculado, de distintas maneras, "con el territorio y los lugares". Y es que, como señala López Rangel (2008: 35), sólo de ese modo podemos identificar y conocer a "los actores sociales que transforman la ciudad", lo cual solo es posible observando su vida cotidiana y los espacios públicos donde transcurre.

En tal sentido, tendríamos que, si bien todo lo relacionado con los procedimientos constructivos $\mathrm{y}$ de diseño tiene una base epistémica, esto es, científica, no puede afirmarse lo mismo si hablamos de experiencias cualitativas y de crítica conceptual. La percepción de un espacio público agradable, o de unas formas arquitectónicas "bellas", lleva a preguntarnos por una teoría del conocimiento que pueda responder por las sensaciones, acciones, percepciones, y otras experiencias subjetivas de las personas en su andar cotidiano por el espacio urbano.

Ya mencioné antes el término teoría social como una corriente de investigación en ciencias 
sociales enfocada a temas tan examinados antes, cuan complicados, como las acciones e interacciones sociales. Uno de los teóricos más importantes de la segunda mitad del siglo anterior, sobre la acción social como un aspecto de la subjetividad en relación con el espacio público y la morfología urbana, ha sido Anthony Giddens, (2006) cuyas aportaciones teóricas son una vía de acceso a nuevas concepciones del conocimiento y la sociedad, en particular la idea de construcción o estructuración (sic) social. En este caso tenemos el concepto contextualidad, entendido como "el carácter situado de una interacción en un espacio-tiempo, que incluye el escenario de una interacción, unos actores copresentes, y una comunicación entre ellos". (Giddens, Ob. Cit.: 394) Éste autor observa una continuidad espacio-temporal donde es "el cuerpo en sus empeños activos con los mundos material y social" (Giddens, Ob. Cit.: 82) de donde surge la percepción, y es el uso de esquemas perceptuales lo que permite elaborar "la temporalidad de una experiencia", como parte sustantiva de la reflexión sobre cualquier acción en general.

Además revisé antes también otro autor, Pierre Bourdieu, (2007) otro investigador multicitado por su gran capacidad para elaborar conceptos en el proceso de ordenamiento del conocimiento sobre la sociedad y la cultura, ha planteado una integración de puntos de vista acerca de los agentes sociales: subjetivo y objetivo.

Para este investigador la corporalidad de las personas, como factor indispensable en el contexto de que se trate, tiene que ver con los conceptos clave heredados de la tradición fenomenológica, el de mundo de vida, término que luego se ajusta como mundo vivido, así como en espacio vivido, (Cfr. Lindón, 2012a: 611) para señalar precisamente las prácticas cotidianas que Bourdieu (2007) llamó sentido práctico, y Giddens (2006) agencia e intencionalidad.

Como mencioné antes, una premisa en éste trabajo es que para comprender y explicar la relación ciudad-agentes sociales, es necesario una crítica de conceptos y un cambio de teorías. Uno de los temas centrales en la epistemología genética de Piaget-García tiene que ver con el cambio de teorías y de categorías conceptuales, y que la reflexión acerca de estos aspectos es cuestión filosófica.

La cuestión conceptual concierne más a las formas de representación que a asuntos de certeza o falsedad; se refiere más bien a la descripción de las relaciones lógicas entre conceptos (Bennett y Hacker,Ob.Cit.: 4-5) ${ }^{3}$, por ejemplo, entrememoria, pensamiento e imaginación, o entre percepción, sensación, y categorización, o entre experiencias subjetivas y eventos neurofisiológicos. Así, el trabajo conceptual no se relaciona con la ciencia empírica sino en la medida que le permite a ésta elucidar los conceptos necesarios para referirse a los recortes teóricos con respecto a la realidad que se va a estudiar.

Por otra parte, ya antes varios estudiosos comprendieron, entre ellos Giddens y Bourdieu, que tanto los científicos sociales como las personas, agentes sociales o actores urbanos, utilizan los mismos recursos cognitivos tales como la conceptualización y el aprendizaje de teorías mediante las prácticas sociales, para comprender el mundo que les rodea.

De tal modo, podemos sostener también que las personas perciben el mundo que les rodea -el espacio edificado- como parte de una construcción de sentido, de significado, en la cual el mundo percibido se manifiesta en términos de objetos distribuidos espacio-temporalmente, es decir, con una continuidad temporal y cognitiva. Lo que afirma la epistemología constructivista es que "la percepción de los objetos como tales, distribuidos en el espacio y con una cierta continuidad en el tiempo, si bien es temprana en los niños, no es innata." (García, 2006: 41)

En tal sentido, existen diferencias fundamentales en el tipo de conocimiento necesario para entender el aprendizaje de categorías conceptuales, y el cambio de conceptos y teorías mismo. Es decir, si como vimos, las experiencias subjetivas de las personas, su vida cotidiana en relación con el espacio público, pueden entenderse como procesos cognitivos que también se identifican con la identidad y la cultura simbólica, con los imaginarios y las representaciones urbanas, etc., tenemos al mismo tiempo distintas formas de entender como sucede ello, lo cual resumí antes como una crítica al dualismo cartesiano y el surgimiento de un nuevo paradigma del conocimiento.

\footnotetext{
${ }^{3}$ Véase Bennett, Maxwell, et al, 2007. Neuroscience \& Philosophy. Brain, Mind, and Languaje. New York, Columbia University Press.
} 
Finalmente, en este nuevo paradigma el conocimiento científico y el humanístico se complementan, ya que mientras podemos entender los procesos de percepción y ordenamiento del espacio mediante un enfoque neurocientífico, éste puede ser allanado utilizando por ejemplo, en cuanto al conocimiento de la actividad simbólica, conceptos ya elaborados en psicoanálisis, la poética, y la historia de las religiones (Cfr. Ricoeur, 2003: 66 y siguientes), además de la arquitectura, claro.

\section{Resultados.}

La nuevas reglas del diseño, que vimos en los ejemplos anteriores de la nueva tradición, la geografía constructivista, y la Teoría Social, tienen un común denominador con otras disciplinas científicas y humanísticas, en las ciencias sociales, y otras de reciente cuño, interesadas en el espacio público y en la vida simbólica de los actores urbanos.

Vimos en primer lugar, que el problema de cómo examinar la vida simbólica o subjetividad de los habitantes urbanos en su relación con el espacio público es correlativo al problema de cómo el conocimiento científico puede explicar o investigar lo que otros piensan, sienten, actúan y perciben. Es decir, se trata del problema de la subjetividad de los actores urbanos y su estatuto epistémico en el conocimiento científico. En tal sentido, las categorías conceptuales mencionadas arriba como instrumentos metodológicos para investigar esta problemática, tales como los imaginarios, representaciones, identidad, cultura simbólica, etc., pueden entenderse como procesos de producción e interpretación de signos, símbolos, y significados, y finalmente como procesos cognitivos de percepción, categorización, conceptualización, e interacción social.

En sentido amplio tenemos dos puntos importantes para la disciplina del diseño: uno acerca del objeto de estudio-la obra arquitectónica y urbana-, y dos, la teorización sobre su conocimiento, que aquí he planteado a partir de una revisión de ciertas categoría conceptuales fundamentales para nuestra disciplina.

Así, como un aspecto del primer punto he señalado el gran cambio ocurrido a partir del modernismo, en el siglo anterior, de las concepciones fundamentales de la arquitectura y del diseño urbano y arquitectónico, en la medida que dicho cambio puede entenderse como parte de otros cambios en el conocimiento, la filosofía, las ciencias y las humanidades. (Mallgrave, 2013; Montaner, ob. Cit.)

Dicho cambio se resume en:

1 crisis del objeto arquitectónico

2 nuevo paradigma del conocimiento

Entre otras consecuencias de ello tenemos el surgimiento de nuevas corrientes de estudio sobre el papel de los actores urbanos en el diseño urbano y arquitectónico, puesto que, en la actualidad, cualquier aspecto de la realidad que queramos examinar es tan complejo, y se encuentra interrelacionado con multitud de otros procesos y planos de realidad, que solamente rebasando las fronteras disciplinares podría abarcarse como tema de estudio.

Y este referente ontológico es por ello mismo el referente de una concepción del mundo ya no como una sucesión de eventos lineales, de causas $\mathrm{y}$ efectos, de formas y de funciones, sino de la idea de que el universo no es lineal, y de que, como señaló Jencks, mas bien avanza a saltos.

En la discusión de los resultados tenemos que, si bien la complejidad de los procesos urbanos surgidos con el modernismo ha requerido enfoques interdisciplinares sobre todo respecto de fenómenos donde se involucra la subjetividad humana, tales como la identidad urbana, los imaginarios, las representaciones sociales, etc., dichos enfoques no son suficientes si sólo se limitan a ofrecer su perspectiva desde su propia frontera disciplinar y metodológica, sino que el reto será establecer metodologías en las cuales se acuerde un problema de estudio y un marco teórico en común.

Un ejemplo de ello lo ofrece la epistemología constructivista, tema ya revisado que se refiere a los tres grandes campos de todo enfoque constructivista: el caso de estudio en particular, o sea, la porción de realidad que el diseñador quiere abarcar en su obra; los procedimientos para su estudio, o metodología, lo que implica una revisión y elucidación conceptual, y un tercero es un eventual cambio de teorías sobre el espacio construido, lo cual tendrá que conducir a un cambio epistémico y filosófico.

Esto quiere decir que antes que todo parece necesario puntualizar cómo se ha dado un cambio en la forma en que las disciplinas científicas organizan el conocimiento. Luego de ello se requiere una crítica conceptual para sustentar 
los cambios en el conocimiento, y en particular del estatuto epistemológico de las categorías conceptuales señaladas, los cuales también pueden ser referidos como esquemas cognitivos de acción, interacción, y representación.

Por ello, tenemos primero que se requiere un doble enfoque, tanto científico como filosófico (o humanístico), mismo que podemos ver concretado en las corrientes de estudios ya señaladas aquí. En particular, el estudio de las subjetividades, representaciones, imaginarios, e interacciones simbólicas, que es también el problema de la acción y atención conjunta, requiere a la vez un enfoque interpretativo desde el cual se da relevancia a la cultura simbólica y al lenguaje narrativo de los individuos, como vimos con Lindón al inicio de la sección 2 (Lindón, Íd.: 31, 33; Lindón, 2011: 21).

Ante ello, en cuanto al papel del diseñador no queda sino interpretar las experiencias fenoménicas de otros mediante sus propias experiencias como habitante de la ciudad. G

\section{Referencias.}

Bennett, M. R., y Hacker, P. M. S., 2007. "Philosophical Foundations of Neuroscience". En: Bennett et al. Neuroscience \& Philosophy. Brain, Mind \& Languaje. New York, Columbia University Press. Pp. 3-48.

Bourdieu, Pierre, 2007. El sentido práctico. Buenos Aires, Siglo XXI Editores.

Davies, Colin, (2011). Reflexiones sobra la arquitectura. Introducción a la teoría arquitectónica. Barcelona, Reverté.

Duhau, Emilio, y Giglia, Angela, 2008. Las reglas del desorden. Habitar la metrópoli. México. UAM Azcapotzalco-Siglo XXI.

Eisenmann, P., (2011). Diez edificios canónicos 1950-2000. Barcelona, Gustavo Gili

Gallagher, Shaun. "Coordinación y creación de sentido en la atención conjunta y la acción conjunta." En: King Dávalos, Patricia, et al. Ciencias cognitivas y filosofia. Entre la cooperación y la integración. Universidad

García, Rolando, 2006. Sistemas Complejos, Barcelona Editorial Gedisa.

Giedion, S., (2009). Espacio, tiempo y arquitectura. Origen y desarrollo de una nueva tradición. Barcelona, Reverté.
Giddens, Anthony, 2006. La constitución de la sociedad. Buenos aires, Amorrurtu.

Lindón, Alicia (2014). "El habitar la ciudad, las redes topológicas del urbanita y la figura del transeúnte". En: Sánchez G., Diego y Domínguez Moreno, L. A. (Coords.). Identidad y Espacio Público. Ampliando ámbitos y practicas. Barcelona, Gedisa. Pp. 55-76.

(2012). "La concurrencia de lo especial y lo social". En: de la Garza Toledo, E. y Leyva, Gustavo (editores). Tratado de metodología de las ciencias sociales: perspectivas actuales. México, Fondo de Cultura-UAM. Pp. 585622)

López Rangel, R., Platas López, F., Romero Fernández, G., y Salceda Salinas, J. U., (2014). La Complejidad y la participación en la producción de Arquitectura y Ciudad. México, UNAM.

López Rangel, R., (2015). "Una vision compleja de los procesos de urbanización de la capital de la República". En: López Rangel, R. y Tena Nuñez, R.A. (coordinadores). Los nuevos paradigmas en los análisis urbanos. Complejidad y urbanización sociocultural en la Ciudad de México. México, UAM-IPN. (Pp. 15-51)

, 2008. "Impensar la ciudad o en busca del pensamiento complejo. Un necesario recorrido epistemológico". En Ramírez Velázquez, Blanca Rebeca (coordinadora). Formas territoriales. Visiones y perspectivas desde la teoría. México, UAM XochimilcoPorrúa. Pp.15-38.

Mallgrave, H. F., 2013. Architecture \& Embodiment. The implications of the new sciences and Humanities for design. Routledge.

Mallgrave, H. F., \& Goodman, D., (2011). An Introduction to Architectural Theory. 1968 to the present. Oxford: Wiley-Blackwell.

Montaner, J. M., (2013), Sistemas arquitectónicos contemporáneos. Barcelona, Ed (2ed). Gustavo Gili.

Montaner, Josep María, 2015. La condición contemporánea de la arquitectura. Barcelona, Gustavo Gili.

Montaner, J. M., (2010), Arquitectura y crítica. Barcelona, Ed (2ed). Gustavo Gili.

Norberg-Schulz, Christian (2005). Los principios de la arquitectura moderna. Barcelona, 
España: Ed. Reverté

Ricoeur, P., (2003). Teoría de la Interpretación.

Discurso y excedente de sentido. México: Siglo XXI.

Tena Núñez, Ricardo A. Ciudad, cultura, y urbanización sociocultural. Conceptos y métodos de análisis urbano. México, Ed. Plaza y Valdés. 2007. 